\title{
GEOMETRIC AND ENERGETIC CRITERIA FOR THE FREE BOUNDARY REGULARITY IN AN OBSTACLE-TYPE PROBLEM
}

\author{
ARSHAK PETROSYAN AND HENRIK SHAHGHOLIAN
}

ABSTRACT. We consider an obstacle-type problem

$$
\begin{array}{ll}
\Delta u=f(x) \chi_{\Omega} & \text { in } \quad D, \\
u=|\nabla u|=0 & \text { on } \quad D \backslash \Omega,
\end{array}
$$

where $D$ is a given open set in $\mathbb{R}^{n}$ and $\Omega$ is an unknown open subset of $D$. The problem originates in potential theory, in connection with harmonic continuation of potentials. The qualitative difference between this problem and the classical obstacle problem is that the solutions here are allowed to change sign. Using geometric and energetic criteria in delicate combination we show the $C^{1,1}$ regularity of the solutions, and the regularity of the free boundary, below the Lipschitz threshold for the right hand side.

\section{INTRODUCTION AND THE MAIN RESUlts}

The statement of the problem. In recent past, starting with the seminal work of Caffarelli [Caf98] and Caffarelli, Karp, and Shahgholian [CKS00], there has been a renewed interest in the study of the well-known obstacle problem, as well as some variations of it. The use of the monotonicity formula of Alt, Caffarelli, and Friedman [ACF84] has been a key ingredient in answering many questions within the free boundary regularity, that was not possible earlier. However, the drawback of using such a strong tool is its limitation in applying it to more general settings.

Our objective in this paper is to find new ways to circumvent such difficulties, as well as to strengthen earlier results, in this direction, to its sharpest possible form. We use Weiss's monotonicity formula [Wei99] in delicate combination with well-known geometric criteria in such problems. This will be explained in details in the text.

The setting we consider here is the obstacle-type problem

$$
\begin{array}{ll}
\Delta u=f(x) \chi_{\Omega} & \text { in } \quad D, \\
u=|\nabla u|=0 & \text { on } \quad D \backslash \Omega,
\end{array}
$$

where $D$ is a given open set in $\mathbb{R}^{n}, \Omega$ is an apriori unknown open subset of $D$, and $u$ is a locally bounded distributional solution.

2000 Mathematics Subject Classification. Primary: 35R35.

Key words and phrases. Obstacle-type problem, $C^{1,1}$ estimates, regularity of free boundary, geometric criterion, energetic criterion, monotonicity formula.

A. Petrosyan is supported in part by NSF grant DMS-0401179. He also thanks the STINT Foundation for supporting his visit to Royal Institute of Technology in Stockholm, Sweden.

H. Shahgholian is partially supported by the Swedish Research Council. The present result is part of the ESF-program GLOBAL. 
For the function $f(x)$ we assume

$$
0<a \leq f(x) \leq b<\infty, \quad x \in D
$$

for some positive constants $a$ and $b$ and

$$
f \in C^{0}(D)
$$

with a modulus of continuity $\omega$, satisfying a certain Dini-type integrability condition, to be specified later. Then we are interested in the optimal regularity of the solution $u$ and the regularity of the free boundary $\partial \Omega \cap D$. Note that we do not make any assumptions on the sign of $u$.

We next give some examples where the problem occurs.

Example 1 (Classical obstacle problem). Given a strictly superharmonic function $\phi \in C_{\text {loc }}^{1,1}(D)$ and a function $g \geq \phi$ on $\partial D$, consider the problem

$$
\int_{D}|\nabla v|^{2} d x \longrightarrow \min , \quad \text { for }\left.\quad v\right|_{\partial D}=g, \quad v \geq \phi \quad \text { in } D .
$$

We may think of the graph of $v$ as a membrane and the graph of $\phi$ as an obstacle. Clearly, the minimizer $v$ is harmonic in the absence of the obstacle. In general, the membrane can touch the obstacle and one will have

$$
\Delta v=\Delta \phi \chi_{\Lambda} \text { in } D,
$$

where $\Lambda:=\{v=\phi\}$ is the coincidence set. Then the difference $u:=v-\phi$ satisfies the conditions (1)-(2) with $f:=-\Delta \phi$ and $\Omega:=\{u>0\}$. We call this problem the classical obstacle problem. We refer to [Caf98] for more details. Note that we have an additional property $u \geq 0$, which comes from the fact that the membrane stays above the obstacle. So, the problem (1)-(2) can be interpreted as the "nosign" version of the problem above, where the membrane is allowed to "cross" the obstacle.

Example 2 (Harmonic continuation of Newtonian potentials). Suppose now we are given a bounded domain $\Omega$ and a function $a \leq f(x) \leq b$ on $\Omega$. Then consider the Newtonian potential generated by the distribution of mass $f \chi_{\Omega}$

$$
U:=\Phi_{n} * f \chi_{\Omega},
$$

where $\Phi_{n}$ is the fundamental solution of the Laplacian in $\mathbb{R}^{n}$. Then $U$ satisfies

$$
\Delta U=-f \chi_{\Omega} \quad \text { a.e. in } \quad \mathbb{R}^{n} .
$$

In particular, $U$ is harmonic outside $\bar{\Omega}$. Suppose now for some $x_{0} \in \partial \Omega$ there exists a harmonic continuation of $U$ through $\partial \Omega$ near $x_{0}$, i.e. a function $v$ such that

$$
\Delta v=0 \quad \text { in } \quad B_{r}\left(x_{0}\right),\left.\quad v\right|_{B_{r}\left(x_{0}\right) \backslash \bar{\Omega}}=U
$$

for some $r>0$. Then, the difference $u:=v-U$ satisfies (1)-(2). Clearly, if $\partial \Omega$ and $f$ are real analytic, such $v$ exists by the Cauchy-Kovalevskaya theorem. So, in a sense, the problem (1)-(2) is an inverse problem for the Cauchy-Kovalevskaya theorem. Note that in this case we have no information on the sign of $u$.

The problem (1)-(2) arises also in inverse source problems [Isa90], in the theory of quadrature domains [Sha92], in Hele-Shaw flow problems [Mar95], in certain models in superconductivity [CSS04] and several other related problems. 
Regularity of solutions and scaling. Let $u \in L_{\text {loc }}^{\infty}(D)$ be a distributional solution of (1). Then $\Delta u \in L^{\infty}(D)$ and we will have

$$
u \in W_{\mathrm{loc}}^{2, p}(D), \quad \text { for every } 1<p<\infty,
$$

by the Calderón-Zygmund estimates. This also implies that

$$
u \in C_{\mathrm{loc}}^{1, \alpha}(D), \quad \text { for every } 0<\alpha<1,
$$

by the Sobolev embedding $W_{\text {loc }}^{2, p} \subset C_{\text {loc }}^{1, \alpha}$ with $\alpha=1-\frac{n}{p}$ for $p>n$. However, it is well known that one cannot generally claim that $u \in W_{\text {loc }}^{2, \infty}=C_{\text {loc }}^{1,1}$, based solely on the local boundedness of $u$ and $\Delta u$. Instead, one can show (e.g. by using the methods in Chapter V of Stein's book [Ste70]) that every directional derivative $v=\partial_{e} u$ is locally of the so-called Zygmund class, which yields the estimate

$$
|\nabla u(x)-\nabla u(y)| \leq C_{K}|x-y| \log \frac{1}{|x-y|},
$$

for any $x, y \in K \subset \subset D$ with $|x-y| \leq 1 / 2$. The presence of the logarithm in this estimate is the source of major complications in this and several other free boundary problems (see e.g. [CGK00], [Bla04], [MW05]), since it does not play well with the natural quadratic scaling of the problem, described next.

Suppose that $u$ is a solution of (1)-(2) and $0 \in \partial \Omega$. For $\lambda>0$ consider the functions

$$
u_{\lambda}(x):=\frac{u(\lambda x)}{\lambda^{2}}, \quad \text { for } \quad x \in D_{\lambda}:=\frac{1}{\lambda} D,
$$

which we call rescalings of $u$. Then $u_{\lambda}$ satisfies

$$
\Delta u_{\lambda}=f_{\lambda}(x) \chi_{\Omega_{\lambda}}, \quad u_{\lambda}=\left|\nabla u_{\lambda}\right|=0 \quad \text { on } \quad D_{\lambda} \backslash \Omega_{\lambda},
$$

where

$$
f_{\lambda}(x):=f(\lambda x), \quad \Omega_{\lambda}:=\frac{1}{\lambda} \Omega .
$$

Note that if $f(x)$ has a modulus of continuity $\omega(r)$, then $f_{\lambda}$ has a modulus of continuity $\omega_{\lambda}(r):=\omega(\lambda r) \leq \omega(r)$, if $0<\lambda<1$. Consider then the family $u_{\lambda}$ of rescalings with small $\lambda>0$. If we had uniform estimates on $u_{\lambda}$, we could extract a converging subsequence $u_{\lambda_{n}} \rightarrow u_{0}$ as $\lambda_{n} \rightarrow 0$, where $u_{0}$ will be a solution of the problem in the entire space $\mathbb{R}^{n}$ (see Section 3 for more details). The problem, however, is that the Zygmund class estimate tells us only that

$$
|u(x)| \leq C_{0}|x|^{2} \log \frac{1}{|x|},
$$

for $x$ near the origin and this estimate does not survive the scaling as $\lambda \searrow 0$. In contrast, if we knew that $u \in C_{\text {loc }}^{1,1}$, we would have the quadratic growth estimate

$$
|u(x)| \leq C_{0}|x|^{2}
$$

for $|x| \leq \delta$ and consequently

$$
\left|u_{\lambda}(x)\right| \leq C_{0}|x|^{2}
$$

for $|x| \leq \delta / \lambda$ and we could perform the above described blowup procedure. Note that $C^{1,1}$ is the highest regularity that one can expect from the solutions $u$ of (1), as $\Delta u$ is discontinuous. The quadratic growth is very well known for the solutions of the classical obstacle problem, see [Caf98], and one only needs $f$ to be bounded measurable. However, when one drops the sign assumption $u \geq 0$, the situation 
changes drastically: the quadratic growth is known only for Lipschitz continuous $f(x)$ (see Theorem 3 and Remark 3 below).

To put the results in this paper into a proper perspective, we start with a brief discussion of some known results, mainly in the case $f=$ const. There are essentially two approaches to the free boundary regularity: geometric, due to L. A. Caffarelli, and energetic, due to G. S. Weiss. But first, let us define the class of local solutions that we will work with.

Definition 1 (Local solutions). Given positive numbers $R$ and $M$ and a modulus of continuity $\omega(r), 0<r<R$, we define $P_{R}^{\omega}(M)$ as the class of distributional solutions $u$ of (1)-(2) in $D=B_{R}$ such that

$$
\begin{aligned}
& |u(x)| \leq M, \quad x \in B_{R}, \\
& |f(x)-f(y)| \leq \omega(|x-y|), \quad x, y \in B_{R}, \\
& f(0)=1, \\
& 0 \in \partial \Omega .
\end{aligned}
$$

Besides, we always require (3). Without loss of generality we assume

$$
\Omega=\Omega_{u}:=D \backslash \Lambda_{u}, \quad \text { where } \Lambda_{u}:=\{u=|\nabla u|=0\} .
$$

We also use $f_{u}$ to indicate the function $f$ that corresponds to the solution $u$.

Remark 1. When $\omega=0$ we obtain the class of solutions $P_{R}^{0}(M)$ with $f=1$. Note that the general case $f=$ const $=: f_{0}>0$ is easily reduced to the normalized case $f=1$ simply by dividing $u$ by $f_{0}$.

Geometric approach. This approach to the free boundary regularity was pioneered by Luis A. Caffarelli in his celebrated papers [Caf77], [Caf80].

Definition 2 (Thickness function). For a solution $u \in P_{R}^{\omega}(M)$ we let

$$
\delta(r, u):=\frac{\min \operatorname{diam}\left(\Lambda_{u} \cap B_{r}\right)}{r},
$$

where $\Lambda_{u}=B_{R} \backslash \Omega$ and min $\operatorname{diam}(E)$ is the minimal diameter of the set $E$, which is the infimum of the distances between two parallel planes that contain the set $E$ in the strip between them.

Theorem 1 (Geometric criterion). Let $u \in P_{1}^{0}(M)$. Then there exists a modulus of continuity $\sigma(r)$, depending only on $M$ and the dimension $n$, such that if

$$
\delta\left(r_{0}, u\right)>\sigma\left(r_{0}\right) \text { for some } 0<r_{0}<1
$$

then

$$
\partial \Omega \cap B_{c_{0}} \quad \text { is an analytic hypersurface }
$$

for some small $c_{0}=c\left(M, r_{0}, n\right)>0$.

Proof. This is Theorem III in Caffarelli, Karp, and Shahgholian [CKS00]. It generalizes Caffarelli's result for the classical obstacle problem, see [Caf98].

Remark 2. Another way of understanding Theorem 1 is that we have the following Caffarelli's alternative, which provides the classification of free boundary points: every $x \in \partial \Omega$ is either

(i) a cusp-like point with a universal estimate $\delta(r, u) \leq \sigma(r)$, or

(ii) a regular point, i.e. the free boundary is regular in a neighborhood of that point. 
Energetic approach. This is a relatively new approach, introduced by Georg S. Weiss in [Wei99].

Definition 3 (Weiss's functional). For a local solution $u \in P_{R}^{\omega}(M)$ consider the following energy functional

$$
W(r ; u, f):=\frac{1}{r^{n+2}} \int_{B_{r}}\left(|\nabla u|^{2}+2 f(x) u\right) d x-\frac{2}{r^{n+3}} \int_{\partial B_{r}} u^{2} d H^{n-1} .
$$

Quite often, when there is no ambiguity, we will abbreviate $W(r ; u, f)$ to $W(r ; u)$.

The functional $W$ has the following remarkable properties:

(1) $W(r ; u)$ is monotone nondecreasing in $r$ for $0<r<R$ if $f=1$, see [Wei99], and "almost" monotone under certain assumptions on $f$ (see Theorem M in Section 2);

(2) If $u \in C_{\mathrm{loc}}^{1,1}$, then $W(0+; u)$ exists and is finite;

(3) If $u \in C_{\text {loc }}^{1,1}$, there are only two possible values for $W(0+; u)$, namely

$$
W(0+; u)= \begin{cases}A_{n}, & \text { and then we call the origin "regular", } \\ 2 A_{n}, & \text { and then we call the origin "singular", }\end{cases}
$$

for some constant $A_{n}>0$, depending only on the dimension $n$.

So, the functional $W$, similarly to Caffarelli's alternative, provides a classification of free boundary points. And "regular" in that classification means regular in the usual sense. The quantitative version of the latter statement is as follows.

Theorem 2 (Energetic criterion). Let $u \in P_{1}^{0}(M)$. Then there exists a modulus of continuity $\sigma(r)$, depending only on $M$ and the dimension $n$, such that if

$$
W\left(r_{0} ; u\right)<2 A_{n}-\sigma\left(r_{0}\right) \quad \text { for some } 0<r_{0}<1
$$

then

$$
\partial \Omega \cap B_{c_{0}} \quad \text { is an analytic hypersurface }
$$

for some small $c_{0}=c\left(M, r_{0}, n\right)>0$.

Proof. This is essentially Theorem 5 in [Wei99]. The main difference is that Weiss does not specify the critical constant, mainly to avoid the classification of homogeneous global solutions (see Lemma 3 in Section 3), which is very specific to this problem and is hard to generalize to a larger class of problems. An alternative proof of this stronger version of Weiss's theorem can be obtained directly from Theorem 1 by utilizing Proposition 1 below.

The following result is a direct link between Theorems 1 and 2: the geometric and energetic conditions in these theorems are equivalent, in a sense, provided the solution is known to be $C^{1,1}$.

Proposition 1 (Equivalence of geometric and energetic criteria for $C^{1,1}$ solutions). Let $u \in P_{1}^{\omega}(M)$ and assume also that $\|u\|_{C^{1,1}\left(B_{1 / 2}\right)} \leq C_{0}$. Then, given $\epsilon>0$, there exist $\eta_{\epsilon}=\eta\left(\epsilon, M, C_{0}, \omega, n\right)>0$ and $r_{\epsilon}=r\left(\epsilon, M, C_{0}, \omega, n\right)>0$ such that

$$
\begin{aligned}
W\left(r_{0} ; u\right)<2 A_{n}-\epsilon & \Rightarrow \delta\left(r_{0}, u\right)>\eta_{\epsilon} \\
\delta\left(r_{0}, u\right)>\epsilon & \Rightarrow W\left(r_{0} ; u\right)<2 A_{n}-\eta_{\epsilon}
\end{aligned}
$$

for any $0<r_{0}<r_{\epsilon}$.

We prove this proposition by a compactness argument in Section 4 . 
$C^{1,1}$ regularity. As we already mentioned, the $C^{1,1}$ estimates are crucial for the study of problem (1)-(2). With no sign assumption on $u$, the largest class of functions $f$ for which these estimates are known is the class of Lipschitz continuous functions.

Theorem 3 ( $C^{1,1}$ regularity when $f \in$ Lip). Let $u \in P_{1}^{\omega}(M)$ with $\omega(r)=K r$. Then, there exists a constant $C_{M}=C(M, K, n)<\infty$ such that

$$
\|u\|_{C^{1,1}\left(B_{1 / 2}\right)} \leq C_{M} .
$$

Proof. This is a particular case of the main result in Shahgholian [Sha03]. A different proof for $f=1$ can be found in [CKS00], which can be also generalized to $f \in$ Lip, see [CS04].

Remark 3. One of the main reasons why the result above is known only for $f \in \operatorname{Lip}$ is as follows. Given a unit vector $e \in \mathbb{R}^{n}$ consider the positive and negative parts of the directional derivative $v_{ \pm}=\left(\partial_{e} u\right)_{ \pm}$. Then they satisfy

$$
\Delta v_{ \pm} \geq-K, \quad v_{ \pm}(0)=0, \quad v_{+} \cdot v_{-}=0 \quad \text { in } \quad B_{1},
$$

where $K$ is the Lip constant of $f$. To such functions one can apply the following deep estimate of Caffarelli, Jerison, and Kenig [CJK02]:

$$
I\left(r, v_{+}\right) I\left(r, v_{-}\right) \leq C\left(1+I\left(1, v_{+}\right)+I\left(1, v_{-}\right)\right)^{2},
$$

for any $0<r<1$, where

$$
I(r, v)=\frac{1}{r^{2}} \int_{B_{r}} \frac{|\nabla v|^{2}}{|x|^{n-2}} d x,
$$

which is a generalization of the celebrated monotonicity formula of Alt, Caffarelli, and Friedman [ACF84]. (The latter says that that $I\left(r, v_{+}\right) I\left(r, v_{-}\right)$is monotone nondecreasing in $r$ if (10) is satisfied with $K=0$.) The application of this estimate is crucial for Shahgholian's method.

Main results. The main objective of this paper is to study the problem (1)-(3) when the $C^{1,1}$ regularity of the solutions is generally unknown. This includes the case of Hölder continuous functions $f$, which will be our main target. So for these $f$, the analogues of Theorems 1 and 2 or the equivalence of their geometric and energetic criteria as in Proposition 1 are unknown. What is interesting, however, is that the combination of the geometric and energetic criteria ensures the $C^{1,1}$ regularity of the solution and implies the regularity of the free boundary. The following theorem is our main result.

Theorem A (Geometric-energetic criterion). Let $u \in P_{1}^{\omega}(M)$ with $\omega$ satisfying

$$
\int_{0} \frac{\omega(\rho) \log \frac{1}{\rho}}{\rho} d \rho<\infty
$$

Then there exists a modulus of continuity $\sigma(r)$ such that if for some $0<r_{0}<1$

$$
\delta\left(r_{0} / 2, u\right) \geq \sigma\left(r_{0}\right) \quad \text { and } \quad W\left(r_{0}, u\right)<2 A_{n}-\sigma\left(r_{0}\right)
$$

then

$$
\|u\|_{C^{1,1}\left(B_{r_{0}}\right)} \leq C_{0}
$$

and

$$
\partial \Omega \cap B_{c_{0}} \quad \text { is a } C^{1} \text { hypersurface }
$$


for some large $C_{0}=C\left(M, r_{0}, \omega, n\right)<\infty$ and small $c_{0}=c\left(M, r_{0}, \omega, n\right)>0$.

Remark 4. The integrability condition (11) is needed for two main reasons: the generalization of Weiss's monotonicity formula (see Section 2) and Blank's sharp version of $C^{1}$ regularity theorem for the classical obstacle problem (see Theorem 4 in Section 6). This condition is slightly stronger than that of Dini, and covers the following cases:

(i) $f \in C^{\alpha}\left(\bar{B}_{1}\right)$ for some $0<\alpha<1$. Then one can take $\omega=C r^{\alpha}$ and condition (11) will be readily satisfied.

(ii) $f \in W^{1, p}\left(B_{R}\right)$ with $p>n$ and some $R>1$, since by the Sobolev embedding theorem $f \in C^{\alpha}\left(\bar{B}_{1}\right)$, with $\alpha=1-\frac{n}{p}$.

Remark 5. Earlier results on the problem with non-Lipschitz $f$ had to impose a certain uniform thickness condition for the zero set of $u$ or a uniform vanishing thinness of the negativity set of $u$ in a whole neighborhood of the point, see [KS99], [BS03]; see also Section 6. The main achievement of Theorem A is that we ask a condition at just one free boundary point.

Finally, we give a purely geometric criterion for the regularity of the free boundary, which is a consequence of Theorem A.

Theorem B (Non-cusp-like points are regular). Let $u \in P_{1}^{\omega}(M)$ with $\omega$ satisfying (11). Then, if

$$
\limsup _{r \searrow 0} \delta(r, u)>0
$$

there exists a small $c_{0}>0$, depending on $u$, such that

$$
\partial \Omega \cap B_{c_{0}} \quad \text { is a } C^{1} \text { hypersurface. }
$$

Remark 6. This theorem essentially provides a weaker version of Caffarelli's alternative (see Remark 2 above): every free boundary point is either

(i) a cusp-like point with $\delta(r, u) \rightarrow 0$ as $r \searrow 0$, or

(ii) a regular point, i.e. the free boundary is regular near that point.

The following corollary from Theorem B is immediate.

Corollary 1 (Exterior cone implies regularity). Let $u \in P_{1}^{\omega}(M)$ with $\omega$ satisfying (11). If $\Omega$ satisfies the exterior cone condition at the origin i.e.

$$
\Omega \cap B_{r_{0}} \subset\left\{x_{n}<K\left|x^{\prime}\right|\right\} \cap B_{r_{0}}
$$

for some constants $r_{0}>0$ and $K<\infty$, after a suitable rotation of coordinate axes, then $\partial \Omega \cap B_{c_{0}}$ is a $C^{1}$ hypersurface for some $c_{0}>0$.

The paper is organized as follows. In Section 2 we prove a generalization of Weiss's monotonicity formula. In Section 3 we recall some known results from the literature, mainly for the case $f=1$. In Section 4 we establish the equivalence of geometric and energetic criteria for $C^{1,1}$ solutions. Sections 5 and 6 contain the proofs of the first and the second parts of Theorem A, respectively, and in Section 7 we prove Theorem B. 


\section{Monotonicity FORMULA}

In this section we establish the following generalization of Weiss's monotonicity formula.

Theorem M (Monotonicity formula). Let $u \in P_{1}^{\omega}(M)$ with $\omega$ satisfying (11). Then there exists a continuous function $F^{\omega}(r)$ for $0 \leq r \leq 1$ with $F^{\omega}(0)=0$ and a constant $C_{M}=C(M, \omega, n)$ such that

$$
W(r ; u, f)+C_{M} F^{\omega}(r) \nearrow \quad \text { for } \quad 0<r<1 / 2 .
$$

Namely, one can take

$$
F^{\omega}(t):=\int_{0}^{t} \omega(\tau) \log \frac{1}{\tau}\left[\frac{\frac{n+3}{n+2}}{\tau}-\frac{\frac{1}{n+2} \tau^{n+1}}{t^{n+2}}\right] d \tau .
$$

Remark 7. Note that when $\omega=0$, i.e. when $f=1$, we have $F^{\omega}=0$ and therefore $W(r ; u, f)$ itself is monotone. This is the original case of Weiss's monotonicity formula. Moreover, in this case something more can be shown: if $W\left(r_{1} ; u\right)=$ $W\left(r_{2} ; u\right)$ for $r_{1}<r_{2}$ then $u$ is homogeneous of degree two in $B_{r_{2}} \backslash B_{r_{1}}$, i.e. $x \cdot \nabla u-$ $2 u=0$ there.

Proof. We are going to exploit the identity

$$
W(\lambda r ; u, f)=W\left(r ; u_{\lambda}, f_{\lambda}\right),
$$

where $u_{\lambda}$ and $f_{\lambda}$ are as in (6)-(8). To proceed, we introduce the following notation for the "homogeneous derivative"

$$
\mathcal{L} w=\left.\frac{d}{d \lambda}\right|_{\lambda=1} w_{\lambda}(x)=x \cdot \nabla w-2 w
$$

for any function $w$, for which it is defined. Note that generally we have

$$
\frac{d}{d \lambda} w_{\lambda}=\frac{1}{\lambda} \mathcal{L} w_{\lambda}
$$

for any $\lambda>0$. We are also going to use the following easily verifiable identities

$$
\begin{gathered}
\mathcal{L}\left(|\nabla w|^{2}\right)=2 \nabla w \cdot \nabla(\mathcal{L} w), \\
\left.\frac{d}{d \lambda}\right|_{\lambda=1}\left(w_{\lambda}\right)^{2}=2 w \mathcal{L} w .
\end{gathered}
$$

Fix now $0<r<s<1 / 2$ and $\sigma=s / r$. Then

$$
\begin{aligned}
W(s ; u, f)- & W(r ; u, f)=W\left(r ; u_{\sigma}, f_{\sigma}\right)-W(r ; u, f) \\
= & \frac{1}{r^{n+2}} \int_{B_{r}}\left(\left|\nabla u_{\sigma}\right|^{2}-|\nabla u|^{2}\right) d x+\frac{2}{r^{n+2}} \int_{B_{r}}\left(f_{\sigma}(x) u_{\sigma}-f(x) u\right) d x \\
& \quad-\frac{2}{r^{n+3}} \int_{\partial B_{r}}\left(u_{\sigma}^{2}-u^{2}\right) d H^{n-1} .
\end{aligned}
$$


Using (14)-(16), we will have

$$
\begin{aligned}
\int_{B_{r}}\left(\left|\nabla u_{\sigma}\right|^{2}-|\nabla u|^{2}\right) d x=\int_{1}^{\sigma} \int_{B_{r}} \frac{1}{\lambda} \mathcal{L}\left(\left|\nabla u_{\lambda}\right|\right)^{2} d x d \lambda \\
=\int_{1}^{\sigma} \frac{2}{\lambda} \int_{B_{r}} \nabla u_{\lambda} \cdot \nabla\left(\mathcal{L} u_{\lambda}\right) d x d \lambda \\
=\int_{1}^{\sigma} \frac{2}{\lambda}\left\{-\int_{B_{r}} \Delta u_{\lambda} \mathcal{L} u_{\lambda} d x+\frac{1}{r} \int_{\partial B_{r}} x \cdot \nabla u_{\lambda} \mathcal{L} u_{\lambda} d H^{n-1}\right\} d \lambda \\
=\int_{1}^{\sigma} \frac{2}{\lambda}\left\{-\int_{B_{r}} f_{\lambda}(x) \mathcal{L} u_{\lambda} d x+\frac{1}{r} \int_{\partial B_{r}} x \cdot \nabla u_{\lambda} \mathcal{L} u_{\lambda} d H^{n-1}\right\} d \lambda
\end{aligned}
$$

and

$$
\int_{\partial B_{r}}\left(u_{\sigma}^{2}-u^{2}\right) d H^{n-1}=\int_{1}^{\sigma} \int_{\partial B_{r}} \frac{2}{\lambda} u_{\lambda} \mathcal{L} u_{\lambda} d H^{n-1} d \lambda .
$$

Then

$$
\begin{aligned}
W(s ; u, f)- & W(r ; u, f) \\
= & \int_{1}^{\sigma} \frac{1}{\lambda}\left\{-\frac{2}{r^{n+2}} \int_{B_{r}} f_{\lambda}(x) \mathcal{L} u_{\lambda} d x+\frac{2}{r^{n+3}} \int_{\partial B_{r}}\left(\mathcal{L} u_{\lambda}\right)^{2} d H^{n-1}\right\} d \lambda \\
& +\frac{2}{r^{n+2}} \int_{B_{r}}\left\{f_{\sigma}(x) u_{\sigma}-f(x) u\right\} d x \\
\geq & \frac{2}{r^{n+2}} \int_{B_{r}}\left\{f(\sigma x) u_{\sigma}-f(x) u-\int_{1}^{\sigma} f(\lambda x)\left(\frac{d}{d \lambda} u_{\lambda}\right) d \lambda\right\} d x .
\end{aligned}
$$

Now that we have this inequality, we argue as follows. Approximate $f$ with a smooth function $f^{\epsilon}$ with the same modulus of continuity $\omega(r)$, for instance by taking a convolution with a mollifier. Then we rewrite

$$
\begin{gathered}
\int_{B_{r}}\left\{f^{\epsilon}(\sigma x) u_{\sigma}-f^{\epsilon}(x) u-\int_{1}^{\sigma} f^{\epsilon}(\lambda x)\left(\frac{d}{d \lambda} u_{\lambda}\right) d \lambda\right\} d x \\
=\int_{B_{r}} \int_{1}^{\sigma}\left(\frac{d}{d \lambda} f^{\epsilon}(\lambda x)\right) u_{\lambda} d \lambda d x \\
=\int_{1}^{\sigma} \int_{B_{r}} x \cdot \nabla f^{\epsilon}(\lambda x) u_{\lambda} d x d \lambda .
\end{gathered}
$$

Next, by changing to polar coordinates and integrating by parts, we transform

$$
\begin{aligned}
\int_{B_{r}} x \cdot \nabla & f^{\epsilon}(\lambda x) u_{\lambda} d x=\int_{\partial B_{1}} \int_{0}^{r} \partial_{\rho} f^{\epsilon}(\lambda \rho \theta) u_{\lambda}(\rho \theta) \rho^{n} d \rho d H_{\theta}^{n-1} \\
= & \int_{\partial B_{1}}\left\{\left[f^{\epsilon}(\lambda r \theta)-1\right] u_{\lambda}(r \theta) r^{n}-\int_{0}^{r}\left[f^{\epsilon}(\lambda \rho \theta)-1\right] \partial_{\rho}\left(u_{\lambda}(\rho \theta) \rho^{n}\right) d \rho\right\} d H_{\theta}^{n-1} .
\end{aligned}
$$

Now, recall that in general we have the Zygmund class estimate

$$
|u| \leq C_{M}|x|^{2} \log \frac{1}{|x|}, \quad|\nabla u| \leq C_{M}|x| \log \frac{1}{|x|}, \quad|x| \leq \frac{1}{2}
$$


for solutions in $P_{1}^{\omega}(M)$. Then we obtain

$$
\begin{aligned}
\int_{B_{r}} x \cdot \nabla f^{\epsilon}(\lambda x) u_{\lambda} d x & \\
& \geq-C_{M}\left(\omega(\lambda r) r^{n+2} \log \frac{1}{\lambda r}+\int_{0}^{r} \omega(\lambda \rho) \rho^{n+1} \log \frac{1}{\lambda \rho} d \rho\right) .
\end{aligned}
$$

Since this inequality is purely in terms of the modulus of continuity $\omega$, we can let $\epsilon \rightarrow 0$ to obtain

$$
\begin{aligned}
W(s ; u, f) & -W(r ; u, f) \\
\geq & -C_{M} \int_{1}^{\sigma}\left(\omega(\lambda r) \log \frac{1}{\lambda r}+\frac{1}{r^{n+2}} \int_{0}^{r} \omega(\lambda \rho) \rho^{n+1} \log \frac{1}{\lambda \rho} d \rho\right) d \lambda .
\end{aligned}
$$

Changing the variables, we can rewrite the above inequality as

$$
\begin{aligned}
W(s ; u, f) & -W(r ; u, f) \\
& \geq-\frac{C_{M}}{r}\left(\int_{r}^{s} \omega(t) \log \frac{1}{t} d t+\int_{r}^{s} \int_{0}^{t} \omega(\tau) \frac{\tau^{n+1}}{t^{n+2}} \log \frac{1}{\tau} d \tau d t\right) \\
& \geq-C_{M} \frac{s}{r}\left(\int_{r}^{s} \frac{\omega(t) \log \frac{1}{t}}{t} d t+\int_{r}^{s} \int_{0}^{t} \omega(\tau) \frac{\tau^{n+1}}{t^{n+3}} \log \frac{1}{\tau} d \tau d t\right) .
\end{aligned}
$$

We also have

$$
\begin{gathered}
\int_{0}^{s} \int_{0}^{t} \omega(\tau) \frac{\tau^{n+1}}{t^{n+3}} \log \frac{1}{\tau} d \tau d t=\int_{0}^{s} \int_{\tau}^{s} \omega(\tau) \frac{\tau^{n+1}}{t^{n+3}} \log \frac{1}{\tau} d t d \tau \\
=\frac{1}{n+2} \int_{0}^{s} \omega(\tau) \tau^{n+1}\left[\frac{1}{\tau^{n+2}}-\frac{1}{s^{n+2}}\right] \log \frac{1}{\tau} d \tau
\end{gathered}
$$

Thus, introducing,

$$
F^{\omega}(t):=\int_{0}^{t} \omega(\tau) \log \frac{1}{\tau}\left[\frac{\frac{n+3}{n+2}}{\tau}-\frac{\frac{1}{n+2} \tau^{n+1}}{t^{n+2}}\right] d \tau
$$

we obtain

$$
W(s ; u, f)-W(r ; u, f) \geq-C_{M} \frac{s}{r}[F(s)-F(r)]
$$

for any $0<r \leq s \leq 1 / 2$. Finally, we can drop the factor $s / r$ in the right-hand side, for instance, by taking a partition $r=s_{0}<s_{1}<\ldots<s_{N}=s$ and applying the inequality for $W\left(s_{i+1} ; u, f\right)-W\left(s_{i} ; u, f\right)$, summing up, and letting the size of the partition tend to 0 . We will arrive at

$$
W(s ; u, f)-W(r ; u, f) \geq-C_{M}[F(s)-F(r)],
$$

for any $0<r \leq s \leq 1 / 2$, which is equivalent to saying that

$$
W(r ; u, f)+C_{M} F(r) \quad \nearrow \quad \text { as } r \nearrow .
$$

The theorem is proved. 


\section{Convergence And Blowup}

In this section we have collected some technical material that will be used later in the paper.

Lemma 1 (Nondegeneracy). Let $u$ be a solution of (1) with $f$ satisfying (3). Then there exists a constant $c_{0}=c(a, b, n)>0$ such that

$$
\sup _{B_{r}(x)} u \geq c_{0} r^{2}
$$

for any free boundary point $x \in \partial \Omega \cap D$ and radius $r>0$ such that $B_{r}(x) \subset D$.

Lemma 2 (Convergence). Let $u_{j} \in P_{1}^{\omega}(M)$ for $j=1,2, \ldots$, and $u_{j} \rightarrow u_{0}$ in $C_{\mathrm{loc}}^{1, \alpha}\left(B_{1}\right)$. Then $u_{0} \in P_{1}^{\omega}(M)$. Moreover, we also have

$$
\operatorname{Int}\left(\Lambda_{u_{0}}\right) \subset \liminf _{j \rightarrow \infty} \operatorname{Int}\left(\Lambda_{u_{j}}\right) .
$$

Proof. Without loss of generality, we may assume that $f_{u_{j}} \rightarrow f_{0}$ for some function $f_{0}$ with the same modulus of continuity $\omega$ as $f_{u_{j}}$ and the same bounds (3). Let now $x \in \Omega_{u_{0}}=B_{1} \backslash \Lambda_{u_{0}}$. Then either $u_{0}$ or $\left|\nabla u_{0}\right|$ are non-zero in a small ball $B_{r}(x)$ and consequently $B_{r / 2}(x) \subset \Omega_{u_{j}}$ for large $j$. Thus, $u_{0}$ will satisfy $\Delta u_{0}=f_{0}$ weakly in $B_{r / 2}(x)$. This shows that $\Delta u_{0}=f_{0}$ in $\Omega_{u_{0}}$. On the other hand, by Calderón-Zygmund estimates, $u_{j}$ are locally uniformly bounded in $W_{\mathrm{loc}}^{2, p}\left(B_{1}\right)$ and therefore also $u_{0} \in W_{\text {loc }}^{2, p}\left(B_{1}\right)$. Since $\nabla u_{0}=0$ on $\Lambda_{u_{0}}$, taking a weak derivative, we will obtain that $D^{2} u_{0}=0$ a.e. on $\Lambda_{u_{0}}$. Thus, we established that $\Delta u_{0}=f_{0} \chi_{\Omega_{u_{0}}}$ weakly in $B_{1}$.

Next, it is immediate that $\left|u_{0}\right| \leq M$ in $B_{1}$. Hence, to conclude that $u_{0} \in P_{1}^{\omega}(M)$, it remains to show that the origin is a free boundary point for $u_{0}$. Clearly $0 \in \Lambda_{u_{0}}$, since $0 \in \Lambda_{u_{j}}, j=1,2, \ldots$. So we have to make sure that the origin is not an interior point of $\Lambda_{u_{0}}$. The latter is a direct consequence from the nondegeneracy (see Lemma 1 above). Indeed, for any $0<r<1$, we have

$$
\sup _{B_{r}} u_{j} \geq c_{0} r^{2}
$$

for all $j$ and consequently

$$
\sup _{B_{r}} u_{0} \geq c_{0} r^{2}
$$

which shows that $B_{r} \not \subset \Lambda_{u_{0}}$. More generally, the same argument also shows the inclusion (17).

Particularly, we can apply the convergence lemma in the following situation. Suppose we have $u \in P_{R}^{\omega}(M)$, which is also known to be in $C^{1,1}\left(B_{r_{0}}\right)$ for some $r_{0}>0$. Then there exists a constant $C$ such that

$$
|u(x)| \leq C|x|^{2}, \quad x \in B_{r_{0}} .
$$

Rescaling, we obtain

$$
\left|u_{\lambda}(x)\right| \leq C|x|^{2}, \quad x \in B_{r_{0} / \lambda},
$$

for any $\lambda>0$, which implies that the family $u_{\lambda}$ is uniformly bounded on compact subsets of $\mathbb{R}^{n}$. Hence, we can extract a subsequence of $u_{\lambda}$ as $\lambda \rightarrow 0$ converging in $C_{\text {loc }}^{1, \alpha}\left(\mathbb{R}^{n}\right)$ to a certain function $u_{0}$, which we call a blowup of $u$ at the origin. This function will be a solution of (1)-(2) with $f=f(0)=1$ in $D=\mathbb{R}^{n}$ or, in other words, it will be a global solution. Moreover, we will have

$$
\left|u_{0}(x)\right| \leq C|x|^{2}, \quad x \in \mathbb{R}^{n},
$$


i.e., $u_{0}$ will have at most quadratic growth at infinity. This motivates the following definition.

Definition 4 (Global solutions with quadratic growth). Given a positive number $M$ we define $P_{\infty}^{0}(M)$ as the class of all solution of (1)-(2) in $D=\mathbb{R}^{n}$ such that

$$
\begin{aligned}
& |u(x)| \leq M\left(1+|x|^{2}\right), \quad x \in \mathbb{R}^{n}, \\
& f(x)=1, \quad x \in \mathbb{R}^{n}, \\
& 0 \in \partial \Omega .
\end{aligned}
$$

The following two examples are of fundamental importance.

Halfspace solutions: $u(x)=h(x)=\frac{1}{2}(x \cdot e)_{+}^{2}$ is a global solution for any unit vector $e \in \mathbb{R}^{n}$.

Polynomial solutions: $u(x)=p(x)=\sum_{i, j=1}^{n} a_{i j} x_{i} x_{j}$ is a global solution, provided $\Delta p=2 \sum_{i=1}^{n} a_{i i}=1$.

Note that both of the examples above are homogeneous of degree 2, i.e.,

$$
u(\lambda x)=\lambda^{2} u(x), \quad x \in \mathbb{R}^{n}, \lambda>0 .
$$

It is a remarkable fact that these are the only homogeneous global solutions.

Lemma 3. Let $u \in P_{\infty}^{0}(M)$ be homogeneous of degree 2. Then $u$ is either a halfspace solution or a polynomial solution.

Proof. This fact is essentially proved in [CKS00]. The proof is by distinguishing the cases when $\operatorname{Int}\left(\Lambda_{u}\right)=\emptyset$ and $\operatorname{Int}\left(\Lambda_{u}\right) \neq \emptyset$ and is based on a deep monotonicity formula of Alt, Caffarelli and Friedman [ACF84]. See Cases 1 and 2 in the proof of Theorem II' in [CKS00]. We also refer to Lemma 6.3 in [CPS04] for a more explicit proof of a generalization of this result to time dependent solutions.

Remark 8. If $u$ is a homogeneous global solution, the energy $W(r ; u)$ is constant in $r$. Thus, without ambiguity we may denote

$$
W(u):=W(r ; u) .
$$

Moreover, if $W(r ; u)=$ const for any global solution $u$ (not necessarily with quadratic growth at infinity), then by Remark 7, $u$ must be a homogeneous solution.

Lemma 4. There exists a constant $A_{n}$, depending only on the dimension $n$, such that for any halfspace solution $h(x)=\frac{1}{2}(x \cdot e)_{+}^{2}$ and any polynomial solution $p(x)=$ $\sum_{i, j=1}^{n} a_{i j} x_{i} x_{j}$, we have

$$
W(h)=A_{n}, \quad W(p)=2 A_{n} .
$$

Moreover, for any global solution $u \in P_{\infty}^{0}(M)$ and $r>0$

$$
A_{n} \leq W(r, u) \leq 2 A_{n}
$$

with a strict inequality from above for all $r>0$, unless $u$ is a polynomial solution.

Remark 9. The case of equality from below in (19) is more delicate: $u$ must be either a halfspace solution or given by $u(x)=\frac{1}{2}(x \cdot e)_{+}^{2}+\frac{1}{2}(x \cdot e+a)_{-}^{2}$ for some $a>0$. 
Proof. Since (18) can already be found in the original paper of Weiss [Wei99], we concentrate here on the rest of the lemma.

We start by observing that the second order derivatives of a global solution $u \in P_{\infty}^{0}(M)$ are uniformly bounded in $\mathbb{R}^{n}$, see Theorem 3 . Then we may consider the rescalings $u_{\lambda}$ and extract converging subsequences as $\lambda \rightarrow 0$ and $\lambda \rightarrow \infty$. We will denote the corresponding limits by $u_{0}$ and $u_{\infty}$. Then

$$
W\left(r ; u_{0}\right)=\lim _{\lambda_{j} \rightarrow 0} W\left(r ; u_{\lambda_{j}}\right)=\lim _{\lambda_{j} \rightarrow 0} W\left(\lambda_{j} r ; u\right)=W(0+; u)=\text { const }
$$

is independent of $r$. Thus, $u_{0}$ is a homogeneous solution and therefore $W(0+; u)$ is either $A_{n}$ or $2 A_{n}$. Similarly,

$$
W\left(r ; u_{\infty}\right)=\lim _{\lambda_{j} \rightarrow \infty} W\left(r ; u_{\lambda_{j}}\right)=\lim _{\lambda_{j} \rightarrow \infty} W\left(\lambda_{j} r ; u\right)=W(\infty ; u)=\text { const }
$$

is independent of $r$ and therefore $W(\infty ; u)$ is either $A_{n}$ or $2 A_{n}$. Hence, by the monotonicity of $W(r ; u)$, we will have

$$
A_{n} \leq W(0+, u) \leq W(r ; u) \leq W(\infty ; u) \leq 2 A_{n},
$$

which proves the required inequality.

Finally, assume that for some $r_{0}>0$ the equality from above is attained, i.e. $W\left(r_{0} ; u\right)=2 A_{n}$. Then, by monotonicity, we must also have $W(r ; u)=2 A_{n}$ for all $r \geq r_{0}$. This will imply that $u$ is homogeneous of degree 2 in $\mathbb{R}^{n} \backslash B_{r_{0}}$, see Remark 7 , and therefore $u=u_{\infty}$ for the blowup $u_{\infty}$ considered above. We will also have $W\left(u_{\infty}\right)=2 A_{n}$, which will imply that $u_{\infty}=p$ is a homogeneous quadratic polynomial. Thus $u=p$ in $\mathbb{R}^{n} \backslash B_{r}$ and by a unique analytic continuation argument we will have that $u=p$ in $\mathbb{R}^{n} \backslash \Lambda_{p}$. Since $\Lambda_{p}$ is nowhere dense, $u=p$ in $\mathbb{R}^{n}$.

\section{Equivalence of geometric And Energetic CRIteria For $C^{1,1}$ SOLUTIONS}

Proof of Proposition 1. 1) First show that

$$
W\left(r_{0} ; u\right)<2 A_{n}-\epsilon \quad \Rightarrow \quad \delta\left(r_{0}, u\right)>\eta_{\epsilon}
$$

if $r_{0}>0$ is sufficiently small. If this is not so, there exist sequences $r_{j} \rightarrow 0$ and $u_{j} \in P_{1}^{\omega}(M), j=1,2, \ldots$, with

$$
\left\|u_{j}\right\|_{C^{1,1}\left(B_{1 / 2}\right)} \leq C_{0}
$$

and such that

$$
W\left(r_{j} ; u_{j}\right)<2 A_{n}-\epsilon \quad \text { and } \quad \delta\left(r_{j}, u_{j}\right) \rightarrow 0 .
$$

Consider then the rescalings

$$
v_{j}:=\left(u_{j}\right)_{r_{j}} \in P_{1 / r_{j}}^{\omega_{r_{j}}}\left(M / r_{j}^{2}\right) .
$$

From $C^{1,1}$ estimates on $u_{j}$ we will have

$$
\left|u_{j}(x)\right| \leq \frac{C_{0}}{2}|x|^{2}, \quad x \in B_{1 / 2}
$$

which will translate to

$$
\left|v_{j}(x)\right| \leq \frac{C_{0}}{2}|x|^{2}, \quad x \in B_{1 /\left(2 r_{j}\right)} .
$$


Thus, we can extract a subsequence $v_{j}$ converging in $C_{\mathrm{loc}}^{1, \alpha}\left(\mathbb{R}^{n}\right)$ to a global solution $v_{0} \in P_{\infty}^{0}\left(C_{0}\right)$. For this global solution we will have

$$
W\left(1 ; v_{0}\right)=\lim _{j \rightarrow \infty} W\left(1 ; v_{j}\right)=\lim _{j \rightarrow \infty} W\left(r_{j} ; u_{j}\right) \leq 2 A_{n}-\epsilon .
$$

On the other hand, we will also have

$$
\delta\left(1, v_{0}\right) \leq \liminf _{j \rightarrow \infty} \delta\left(1, v_{j}\right)=\liminf _{j \rightarrow \infty} \delta\left(r_{j}, u_{j}\right)=0
$$

This means $\Delta v_{0}=1$ a.e. in $B_{1}$. Therefore, any blowup of $v_{0}$ at the origin must also have that property, which is possible only if the blowup is a quadratic polynomial (by Liouville's theorem). Thus,

$$
W\left(0+; v_{0}\right)=2 A_{n}
$$

which contradicts to the fact that

$$
W\left(0+; v_{0}\right) \leq W\left(1 ; v_{0}\right) \leq 2 A_{n}-\epsilon
$$

2) Next we show that

$$
\delta\left(r_{0}, u\right)>\epsilon \quad \Rightarrow \quad W\left(r_{0} ; u\right)<2 A_{n}-\eta_{\epsilon}
$$

for sufficiently small $r_{0}>0$. Assuming the contrary, we will have sequences $r_{j} \rightarrow 0$ and $u_{j} \in P_{1}^{\omega}(M), j=1,2, \ldots$, with

$$
\left\|u_{j}\right\|_{C^{1,1}\left(B_{1 / 2}\right)} \leq C_{0}
$$

and such that

$$
\delta\left(r_{j}, u_{j}\right) \geq \epsilon \quad \text { and } \quad \liminf _{j \rightarrow \infty} W\left(r_{j} ; u_{j}\right) \geq 2 A_{n}
$$

As before, consider the rescalings

$$
v_{j}:=\left(u_{j}\right)_{r_{j}} \in P_{1 / r_{j}}^{\omega_{r_{j}}}\left(M / r_{j}^{2}\right)
$$

which will also satisfy

$$
\left|v_{j}(x)\right| \leq \frac{C_{0}}{2}|x|^{2}, \quad x \in B_{1 /\left(2 r_{j}\right)}
$$

and extract a subsequence $v_{j}$ converging in $C_{\mathrm{loc}}^{1, \alpha}\left(\mathbb{R}^{n}\right)$ to a global solution $v_{0} \in$ $P_{\infty}^{0}\left(C_{0}\right)$. We will have

$$
W\left(1 ; v_{0}\right)=\lim _{j \rightarrow \infty} W\left(1 ; v_{j}\right)=\lim _{j \rightarrow \infty} W\left(r_{j}, u_{j}\right) \geq 2 A_{n}
$$

Invoking Lemma 4 , we obtain that $W\left(1 ; v_{0}\right)=2 A_{n}$, which is possible only if $v_{0}$ is a quadratic polynomial. But this contradicts to

$$
\delta\left(1, v_{0}\right) \geq \limsup _{j \rightarrow \infty} \delta\left(1, v_{j}\right)=\limsup _{j \rightarrow \infty} \delta\left(r_{j}, u_{j}\right) \geq \epsilon>0 .
$$

The proof is complete. 


\section{5. $C^{1,1}$ REGULARITY}

In the next two sections we prove Theorem A by splitting it into two parts. The main result of this section, Theorem A1, is equivalent to the first part of Theorem A.

Before we proceed, note that from now on we will not explicitly indicate the dependence of constants on the dimension $n$ and/or on the modulus of continuity $\omega ;$ this should be clear from the context.

Theorem $\mathbf{A} 1$ ( $C^{1,1}$ regularity). Let $u \in P_{1}^{\omega}(M)$ with $\omega$ satisfying (11). Given $\epsilon>0$, there exists $r_{\epsilon, M}>0$ such that if for some $0<r_{0}<r_{\epsilon, M}$

$$
\delta\left(r_{0} / 2, u\right) \geq \epsilon \quad \text { and } \quad W\left(r_{0}, u\right)<2 A_{n}-\epsilon
$$

then

$$
\|u\|_{L^{\infty}\left(B_{r}\right)} \leq C_{\epsilon} r^{2} \quad \text { for every } \quad 0<r \leq r_{0}
$$

and

$$
\|u\|_{C^{1,1}\left(B_{c_{0}}\right)} \leq C_{\epsilon, M, r_{0}}
$$

for some small $c_{0}=c\left(\epsilon, M, r_{0}\right)>0$.

We start with a growth estimate near the origin.

Lemma 5. Let $u \in P_{1}^{\omega}(M)$. Given $\epsilon>0$ and $W_{0}<\infty$, there exist $\lambda_{\epsilon, W_{0}}>0$ and $C_{\epsilon, W_{0}}<\infty$ such that if for some $0<r_{0}<\lambda_{\epsilon, W_{0}}$

$$
\delta\left(r_{0} / 2, u\right)>\epsilon \quad \text { and } \quad W\left(r_{0} ; u\right) \leq W_{0}
$$

then

$$
\|u\|_{L^{\infty}\left(B_{r_{0} / 2}\right)} \leq\left(C_{\epsilon, W_{0}}\right) r_{0}^{2} .
$$

Remark 10. We emphasize that the constants $\lambda_{\epsilon, W_{0}}$ and $C_{\epsilon, W_{0}}$ do not depend on $M$.

Proof. Note that it will suffice to prove that

$$
\|u\|_{L^{2}\left(\partial B_{r_{0}}\right)} \leq\left(C_{\epsilon, W_{0}}\right) r_{0}^{n+1},
$$

since one has an interior estimate

$$
\|u\|_{L^{\infty}\left(B_{r_{0} / 2}\right)} \leq C_{0}\left(\frac{1}{r_{0}^{n-1}}\|u\|_{L^{2}\left(\partial B_{r_{0}}\right)}+\|f\|_{L^{\infty}\left(B_{r_{0}}\right)} r_{0}^{2}\right) .
$$

This can be easily seen by decomposing $u$ in $B_{r_{0}}$ into the sum of a harmonic function with the same boundary values and a function with zero boundary values and the same Laplacian.

We argue by contradiction: assume that there exist sequences $r_{j} \searrow 0$ and $u_{j} \in$ $P_{1}^{\omega}\left(M_{j}\right)$ such that

$$
\begin{aligned}
& \delta\left(r_{j} / 2, u_{j}\right)>\epsilon, \\
& W\left(r_{j} ; u_{j}\right) \leq W_{0}, \\
& C_{j}:=\frac{\left.\left\|u_{j}\right\|_{L^{2}\left(\partial B_{r_{j}}\right.}\right)}{r_{j}^{n+1}} \rightarrow \infty
\end{aligned}
$$

Consider then the functions

$$
w_{j}(x):=\frac{u_{j}\left(r_{j} x\right)}{C_{j} r_{j}^{2}}, \quad x \in B_{1 / r_{j}} .
$$


Then $w_{j}$ will be a solution of (1) with

$$
\tilde{f}_{j}(x):=\frac{f_{u_{j}}\left(r_{j} x\right)}{C_{j}}, \quad x \in B_{1 / r_{j}} .
$$

Besides, we will have

$$
\begin{aligned}
& \delta\left(1 / 2, w_{j}\right) \geq \epsilon \\
& \left\|w_{j}\right\|_{L^{2}\left(\partial B_{1}\right)}=1 .
\end{aligned}
$$

Furthermore,

$$
W\left(1 ; w_{j}, \tilde{f}_{j}\right)=\frac{W\left(r_{j} ; u_{j}, f_{u_{j}}\right)}{C_{j}^{2}} \leq \frac{W_{0}}{C_{j}^{2}}
$$

In particular,

$$
\int_{B_{1}}\left|\nabla w_{j}\right|^{2} \leq 2 \int_{\partial B_{1}} w_{j}^{2}+\frac{W_{0}}{C_{j}^{2}}+\frac{1}{C_{j}} \sup _{B_{r_{j}}} f_{u_{j}} .
$$

Since the last two terms converge to 0 , the right-hand side is bounded and we conclude that $w_{j}$ are uniformly bounded in $W^{1,2}\left(B_{1}\right)$. Hence, over a subsequence

$$
w_{j} \rightarrow w_{0} \quad \text { weakly in } \quad W^{1,2}\left(B_{1}\right) .
$$

Now the compactness of the Sobolev trace operator implies that, over yet another subsequence,

and consequently

$$
w_{j} \rightarrow w_{0} \quad \text { strongly in } \quad L^{2}\left(\partial B_{1}\right)
$$

$$
\left\|w_{0}\right\|_{L^{2}\left(\partial B_{1}\right)}=1 \text {. }
$$

On the other hand, passing to the limit in (24), we will obtain

$$
\int_{B_{1}}\left|\nabla w_{0}\right|^{2} \leq 2 \int_{\partial B_{1}} w_{0}^{2}
$$

We will also have

$$
\left|\Delta w_{j}\right| \leq \sup _{B_{1}} \tilde{f}_{j} \leq \frac{1}{C_{j}} \sup _{B_{r_{j}}} f_{u_{j}} \rightarrow 0 \quad \text { in } \quad B_{1},
$$

implying that $w_{0}$ is harmonic in $B_{1}$. On the other hand, by Almgren's frequency lemma (e.g. see Lemma 4.1 in [Wei01])

$$
\int_{B_{1}}\left|\nabla w_{0}\right|^{2} \geq 2 \int_{\partial B_{1}} w_{0}^{2}
$$

since $w_{0}(0)=\left|\nabla w_{0}(0)\right|=0$, and the inequalities (26) and (28) are possible iff $w_{0}$ is a homogeneous quadratic polynomial in $B_{1}$. Now, to complete the proof, we note that

$$
\delta\left(1 / 2, w_{0}\right) \geq \epsilon .
$$

This follows from (22) and the fact that, over a subsequence, $w_{j} \rightarrow w_{0}$ in $C_{\text {loc }}^{1, \alpha}\left(B_{q}\right)$, since $w_{j}$ and $\Delta w_{j}$ are locally uniformly bounded in $B_{1}$. Thus, $w_{0}$ must be identically 0 , contradicting (25).

To simplify the statement of the next few lemmas, we use the notion of $\epsilon$-closeness of two functions.

Definition 5. ( $\epsilon$-closeness) We say that functions $u$ and $v$ are $\epsilon$-close on a set $E$, whenever $\|u-v\|_{L^{\infty}(E)}<\epsilon$. We also use $\operatorname{dist}(u, v)$ on $E$ to indicate $\|u-v\|_{L^{\infty}(E)}$. 
Lemma 6. Let $v \in P_{1 / 4}^{0}\left(C_{0}\right)$ and $W(1 / 4 ; v) \leq 2 A_{n}-\epsilon / 2$. Then for every $\beta_{0}>0$ there exists a small $t_{0}=t_{\epsilon, \beta_{0}, C_{0}}>0$ such that $v$ is $\beta_{0} t_{0}^{2}$-close to a halfspace solution in $B_{t_{0}}$.

Proof. Assume the contrary. Then there exist sequences $t_{j} \searrow 0$ and $v_{j} \in P_{1 / 4}^{0}\left(C_{0}\right)$ such that

$$
\operatorname{dist}\left(v_{j}, h\right) \geq \beta_{0} t_{j}^{2} \quad \text { in } \quad B_{t_{j}},
$$

for any halfspace solution $h$. Consider now the rescalings

$$
w_{j}:=\left(v_{j}\right)_{t_{j}} \in P_{1 / 4 t_{j}}^{0}\left(C_{0} / t_{j}^{2}\right) .
$$

Using the $C^{1,1}$ estimates, available for solutions with $f=1$, see Theorem 3 , we can extract a subsequence $w_{j} \rightarrow w_{0}$ converging in $C_{\mathrm{loc}}^{1, \alpha}\left(\mathbb{R}^{n}\right)$ to a global solution $w_{0} \in P_{\infty}^{0}\left(C\left(C_{0}\right)\right)$. Note that from construction we will have

$$
\begin{aligned}
W\left(r ; w_{0}\right) & =\lim _{j \rightarrow \infty} W\left(r ; w_{j}\right)=\lim _{j \rightarrow \infty} W\left(r t_{j} ; v_{j}\right) \\
& \leq \lim _{j \rightarrow \infty} W\left(1 / 4 ; v_{j}\right) \leq 2 A_{n}-\epsilon / 2,
\end{aligned}
$$

for any $r>0$. In particular, $W\left(\infty ; w_{0}\right)<2 A_{n}$. Repeating the arguments as in the proof of Lemma 4 , we find that $W\left(\infty ; w_{0}\right)$ can take only two values: $A_{n}$ or $2 A_{n}$. Hence, $W\left(\infty ; w_{0}\right)=A_{n}$, which implies that $W\left(r ; w_{0}\right)=A_{n}$ for any $r>0$. The latter is possible only if $w_{0}$ is a halfspace solution, see Remark 8 and Lemma 4. But then

$$
\operatorname{dist}\left(w_{j}, w_{0}\right)<\beta_{0} \quad \text { in } \quad B_{1}
$$

for large $j$, implying that

$$
\operatorname{dist}\left(v_{j}, w_{0}\right)<\beta_{0} t_{j}^{2} \quad \text { in } \quad B_{t_{j}} .
$$

This contradicts (29).

Lemma 7. Let $u \in P_{1}^{\omega}(M)$. Given $\epsilon>0$ and $\beta_{0}>0$ there exist small $\mu_{\epsilon, \beta_{0}}>0$ and $t_{\epsilon, \beta_{0}}>0$ such that if for some $0<r_{0}<\mu_{\epsilon, \beta_{0}}$

$$
\delta\left(r_{0} / 2, u\right) \geq \epsilon, \quad W\left(r_{0} / 4 ; u\right)<2 A_{n}-\epsilon / 2, \quad W\left(r_{0} ; u\right)<2 A_{n}-\epsilon / 2
$$

then

$$
u \text { is } \beta_{0}\left(r_{0} t_{\epsilon, \beta_{0}}\right)^{2} \text {-close to a halfspace solution in } B_{r_{0} t_{\epsilon, \beta_{0}}} \text {. }
$$

Remark 11. The double energetic condition is necessary to avoid the use of the monotonicity formula and the dependence on constant $M$. The latter is crucial in our arguments.

Proof. Let $t_{\epsilon, \beta_{0}}=t_{\epsilon, \beta_{0} / 2, C_{0}}$ be as in Lemma 6 above with $C_{0}=C_{\epsilon, 2 A_{n}}$ as in Lemma 5 and assume that the statement of Lemma 7 fails. Then there exist sequences $r_{j} \searrow 0$ and $u_{j} \in P_{1}^{\omega}\left(M_{j}\right)$ such that

$$
\delta\left(r_{j} / 2, u_{j}\right) \geq \epsilon, \quad W\left(r_{j} / 4 ; u_{j}\right)<2 A_{n}-\epsilon / 2, \quad W\left(r_{j} ; u_{j}\right)<2 A_{n}-\epsilon / 2
$$

and

$$
\operatorname{dist}\left(u_{j}, h\right) \geq \beta_{0}\left(t_{\epsilon, \beta_{0}} r_{j}\right)^{2} \quad \text { in } \quad B_{t_{\epsilon, \beta_{0}} r_{j}},
$$

for any halfspace solution $h$. Consider then the rescalings

$$
v_{j}:=\left(u_{j}\right)_{r_{j}} \in P_{1 / r_{j}}^{\omega_{r_{j}}}\left(M_{j} / r_{j}^{2}\right) .
$$


We will have that

$$
\begin{aligned}
\delta\left(1 / 2, v_{j}\right) & \geq \epsilon, \\
\operatorname{dist}\left(v_{j}, h\right) & \geq \beta_{0} t_{\epsilon, \beta_{0}}^{2} \quad \text { in } \quad B_{t_{\epsilon, \beta_{0}}},
\end{aligned}
$$

for any halfspace solution $h$. Moreover, by Lemma 5 , we will have that

$$
\left|u_{j}\right| \leq\left(C_{\epsilon, 2 A_{n}}\right) r_{j}^{2} \quad \text { in } \quad B_{r_{j} / 2}
$$

which is equivalent to

$$
\left|v_{j}\right| \leq C_{\epsilon, 2 A_{n}} \quad \text { in } \quad B_{1 / 2} .
$$

Thus, we can extract a subsequence $v_{j} \rightarrow v_{0}$, converging in $C^{1, \alpha}$ norm in $B_{1 / 4}$. The limiting $v_{0}$ will be a solution with $f=1$. Besides, we will also have

$$
W\left(1 / 4 ; v_{0}\right)=\lim _{j \rightarrow \infty} W\left(1 / 4 ; v_{j}\right)=\lim _{j \rightarrow \infty} W\left(r_{j} / 4 ; u_{j}\right) \leq 2 A_{n}-\epsilon / 2 .
$$

Applying now Lemma 6 above, we obtain that $v_{0}$ is $\left(\beta_{0} / 2\right)\left(t_{\epsilon, \beta_{0}}\right)^{2}$-close to a halfspace solution in $B_{t_{\epsilon, \beta_{0}}}$. But then $v_{j}$ is $\left(3 \beta_{0} / 4\right)\left(t_{\epsilon, \beta_{0}}\right)^{2}$-close to a halfspace solution in $B_{t_{\epsilon, \beta_{0}}}$, for sufficiently large $j$, which is a contradiction. This proves the lemma.

Lemma 8. Let $u \in P_{1}^{\omega}(M)$ be $\beta_{0}$-close to a halfspace solution in $B_{1}$. Then, if $\beta_{0}$ is sufficiently small (independent of $M$ ), we will have

$$
\delta(u, 1 / 2)>1 / 2 \text {. }
$$

Proof. Assume the contrary. Then there exist a sequence $u_{j} \in P_{1}^{\omega}\left(M_{j}\right)$ and halfspace solutions $h_{j}$ such that

$$
\operatorname{dist}\left(u_{j}, h_{j}\right) \rightarrow 0 \quad \text { in } \quad B_{1}
$$

and

$$
\delta\left(u_{j}, 1 / 2\right) \leq 1 / 2, \quad j=1,2, \ldots
$$

Without loss of generality we may assume that $h_{j} \rightarrow h_{0}$ which will imply that, over a subsequence, $u_{j} \rightarrow h_{0}$ in $C_{\mathrm{loc}}^{1, \alpha}$ in $B_{1}$. But then, we will obtain that

$$
\delta\left(u_{j}, 1 / 2\right) \rightarrow \delta\left(h_{0}, 1 / 2\right)=1
$$

by Lemma 2 . This is clearly a contradiction.

Proof of Theorem A1.

Step 1: We start with an observation that by the monotonicity formula (Theorem M) there exists $\rho_{\epsilon, M}>0$ such that

$$
W\left(r_{1} ; u\right) \leq W\left(r_{2} ; u\right)+\epsilon / 2, \quad \text { for every } 0<r_{1} \leq r_{2} \leq \rho_{\epsilon, M},
$$

if $u \in P_{1}^{\omega}(M)$. In particular,

$$
W\left(r_{0} ; u\right)<2 A_{n}-\epsilon \text { for some } 0<r_{0}<\rho_{\epsilon, M}
$$

implies

$$
W(r ; u) \leq 2 A_{n}-\epsilon / 2 \text { for every } 0<r \leq r_{0} .
$$

Step 2: Let $\beta_{0}$ be as in Lemma $8, t_{\epsilon}=t_{\epsilon, \beta_{0}}$ and $\mu_{\epsilon}=\mu_{\epsilon, \beta_{0}}$ as in Lemma 7 and $\lambda_{\epsilon}=\lambda_{\epsilon, 2 A_{n}}$ as in Lemma 5. Assume now that

$$
\delta\left(r_{0} / 2, u\right) \geq \epsilon \quad \text { and } \quad W\left(r_{0} ; u\right) \leq 2 A_{n}-\epsilon
$$

for some

$$
0<r_{0}<\min \left\{\lambda_{\epsilon}, \mu_{\epsilon}, \rho_{\epsilon, M}\right\}
$$


Then we also have $W\left(r_{0} / 4 ; u\right) \leq 2 A_{n}-\epsilon / 2$, see Step 1 . Then by Lemma 7 , we will have that

$$
u \text { is } \beta_{0}\left(r_{0} t_{\epsilon}\right)^{2} \text {-close to a halfspace solution in } B_{r_{0} t_{\epsilon}} .
$$

Applying now Lemma 8 to the rescaling $u_{r_{0} t_{\epsilon}}$, we find that

$$
\delta\left(r_{0} t_{\epsilon} / 2, u\right)=\delta\left(1 / 2, u_{r_{0} t_{\epsilon}}\right)>1 / 2 .
$$

Step 3: Induction. Set now

$$
u^{(k)}(x)=u_{t_{\epsilon}^{k}}(x)=\frac{u\left(t_{\epsilon}^{k} x\right)}{t_{\epsilon}^{2 k}}, \quad k=0,1,2, \ldots
$$

Then from Steps 1 and 2, we have that

$$
\begin{gathered}
W\left(r ; u^{(1)}\right)=W\left(t_{\epsilon} r ; u\right) \leq 2 A_{n}-\epsilon / 2 \text { for every } 0<r \leq r_{0}, \\
\delta\left(r_{0} / 2, u^{(1)}\right)=\delta\left(r_{0} t_{\epsilon} / 2, u\right) \geq 1 / 2>\epsilon .
\end{gathered}
$$

Then we can apply Step 2 to the function $u^{(1)}$ to obtain

$$
\delta\left(r_{0} t_{\epsilon} / 2, u^{(1)}\right) \geq 1 / 2 .
$$

Iterating this process, we obtain that for any $k=1,2, \ldots$

$$
\delta\left(r_{0} t_{\epsilon}^{k} / 2, u\right) \geq 1 / 2 .
$$

Then, applying Lemma 5, we obtain

$$
\|u\|_{L^{\infty}\left(B_{r_{0} t_{\epsilon}^{k} / 2}\right)} \leq C_{\epsilon}\left(r_{0} t_{\epsilon}^{k}\right)^{2}, \quad k=1,2, \ldots
$$

By a standard procedure, this implies that

$$
\|u\|_{L^{\infty}\left(B_{r}\right)} \leq \tilde{C}_{\epsilon} r^{2}, \quad \text { for every } \quad 0<r \leq r_{0} .
$$

This proves (20).

Step 4: Finally, to conclude the proof, we notice the following. If instead of the origin we take any of the free boundary points $x_{0} \in \partial \Omega \cap B_{c_{0}}$, for some small constant $c_{0}=c_{\epsilon, M, r_{0}}>0$, and define

$$
\tilde{u}:=\frac{u\left(\cdot+x_{0}\right)}{f\left(x_{0}\right)} \in P_{1 / 2}^{2 \omega}(2 M)
$$

we will have that

$$
\delta\left((3 / 4) r_{0}, \tilde{u}\right) \geq \epsilon / 2, \quad W\left(r_{0}, \tilde{u}\right) \leq \frac{2 A_{n}-\epsilon / 2}{f\left(x_{0}\right)^{2}} \leq 2 A_{n}-\epsilon / 4
$$

(Note that $\left|1-f\left(x_{0}\right)\right| \leq \omega\left(c_{0}\right)$ can be made as small as we wish). This is slightly different than the condition we had for $u$, nevertheless, we can easily adjust the lemmas above to deal with these modified conditions. Then, going back to the original $u$, we will obtain that it satisfies

$$
|u(x)| \leq C_{0} \operatorname{dist}(x, \partial \Omega)^{2}, \quad \text { for any } \quad x \in B_{c_{0}} .
$$

Invoking now a standard procedure based on the interior derivative estimates, see e.g. [CKS00], we establish (21) and thereby conclude the proof of the theorem. 


\section{REgularity OF THE FREE BOUNDARY}

In this section we prove the second half of Theorem A.

Theorem A2 (Regularity of the free boundary). Let $u \in P_{1}^{\omega}(M)$ with $\omega$ satisfying (11). Given $\epsilon>0$, there exists $r_{\epsilon, M}>0$ such that if for some $0<r_{0}<r_{\epsilon, M}$

$$
\delta\left(r_{0} / 2, u\right) \geq \epsilon \quad \text { and } \quad W\left(r_{0}, u\right)<2 A_{n}-\epsilon
$$

then

$$
\partial \Omega \cap B_{c_{0}} \quad \text { is a } C^{1} \text { hypersurface }
$$

for some small $c_{0}=c\left(\epsilon, M, r_{0}\right)>0$.

A similar result was known earlier under much stronger assumptions on $u$, such as the uniform thickness of $\Lambda_{u}$ in a neighborhood of the origin, i.e.

$$
\frac{\min \operatorname{diam}\left(\Lambda_{u} \cap B_{r}\left(x_{0}\right)\right)}{r} \geq \delta_{0}>0,
$$

for any $0<r<r_{0}$ and $x_{0} \in B_{\rho_{0}} \cap \partial \Omega$, see Blank and Shahgholian [BS03], or the uniform vanishing thinness of the negativity set $\Omega^{-}=\{u<0\}$, i.e.

$$
\lim _{r \searrow 0} \frac{\max \operatorname{rad}\left(\Omega^{-} \cap B_{r}\left(x_{0}\right)\right)}{r}=0,
$$

uniformly in $x_{0} \in B_{\rho_{0}} \cap \partial \Omega$, see Karp and Shahgholian [KS99]. (Here max $\operatorname{rad}(E)$ is the maximal radius of the set $E$, which is the supremum of the radii of balls fully contained in $E$.)

Our geometric-energetic condition implies both of the conditions above. However, we choose to work with the former one, since it yields a slightly stronger result.

The main idea of the proof is to show that $u$ becomes nonnegative in a small neighborhood of $u$. Then the result will follow from the corresponding result for the classical obstacle problem. Namely, we will use Blank's sharp form for the $C^{1}$ regularity of the free boundary, see [Bla01] and Theorem 4 below.

Lemma 9 (Local nonnegativity). Let $u \in P_{1}^{\omega}(M)$ with $\omega$ satisfying (11). Given $\epsilon>0$, there exists $r_{\epsilon, M}>0$ such that if for some $0<r<r_{\epsilon, M}$

$$
\delta\left(r_{0} / 2, u\right) \geq \epsilon \quad \text { and } \quad W\left(r_{0}, u\right)<2 A_{n}-\epsilon
$$

then there exists $\rho_{\epsilon, M, r_{0}}>0$ such that $u \geq 0$ in $B_{\rho_{\epsilon, M, r_{0}}}$.

Proof. We go back to the proof of Theorem A1, where we showed that

$$
\delta\left(t_{\epsilon}^{k} r_{0} / 2, u\right) \geq 1 / 2, \quad \text { for } \quad k=1,2, \ldots
$$

This immediately implies that

$$
\delta(r / 2, u) \geq t_{\epsilon} / 2, \quad \text { for } \quad 0<r<r_{0},
$$

by finding the integer $k$ such that $t_{\epsilon}^{k+1} r_{0} \leq r<t_{\epsilon}^{k} r_{0}$.

Arguing now as in Step 4 of the proof of Theorem A1, we find that

$$
\delta_{x_{0}}(r, u):=\frac{\min \operatorname{diam}\left(\Lambda_{u} \cap B_{r}\left(x_{0}\right)\right)}{r} \geq \tilde{t}_{\epsilon} / 2,
$$

for any $0<r<r_{0}$ and $x_{0} \in B_{c_{0}} \cap \partial \Omega$, or in other words $\Lambda_{u}$ is uniformly thick in a neighborhood of the origin. Then the lemma follows from Theorem 1.6 of Blank and Shahgholian [BS03]. 
The following theorem of Blank is the sharp version of Caffarelli's theorem for (nonnegative) solutions of the classical obstacle problem.

Theorem 4. Let $u \in P_{1}^{\omega}(M), u \geq 0$, with $\omega$ satisfying the Dini condition

$$
\int_{0} \frac{\omega(\rho)}{\rho} d \rho<\infty
$$

Then there exists a modulus of continuity $\sigma(r)$ such that if

$$
\delta\left(r_{0}, u\right)>\sigma\left(r_{0}\right) \quad \text { for some } 0<r_{0}<1
$$

then

$$
\partial \Omega \cap B_{c_{0}} \quad \text { is a } C^{1} \text { hypersurface }
$$

for some small $c_{0}=c\left(M, r_{0}\right)>0$.

Proof. See Theorem 0.1 in [Bla01].

We are now ready to prove the main result of this section.

Proof of Theorem A2. By Lemma 9, $u \geq 0$ in $\rho_{\epsilon, M, r_{0}}$-neighborhood of the origin. Since the modulus $\omega$ satisfies condition (11) which is stronger than the Dini integrability condition, we can apply Theorem 4 . To finish the proof, we must find $\hat{r}_{0}>0$ such that $\delta\left(\hat{r}_{0}, u\right)>\sigma\left(\hat{r}_{0}\right)$ for the modulus of continuity $\sigma(r)$ as in Theorem 4 . But the latter follows easily from the fact that $\delta(r / 2, u) \geq t_{\epsilon} / 2>0$ for all $0<r<r_{0}$, as we established in the proof of Lemma 9.

This completes the proof of the theorem.

\section{Purely GeOMETRIC CRITERION}

In this section we prove Theorem $\mathrm{B}$, which provides a purely geometric criterion for the regularity of the free boundary.

Proof of Theorem B. Let $u \in P_{1}^{\omega}(M)$ and $r_{j} \rightarrow 0$ be a sequence such that

$$
\delta\left(r_{j} / 2, u\right) \geq \epsilon>0, \quad j=1,2, \ldots
$$

We claim that

$$
W(0+; u)=\lim _{r \searrow 0} W(r ; u)=A_{n} .
$$

(Note that the limit exists by Theorem M). To prove this claim, observe that $u \in P_{1}^{\omega}(M)$ automatically implies that

$$
W(1 / 2 ; u) \leq W_{M} .
$$

Then, by the monotonicity formula (Theorem A) we can assume also that

$$
W(r ; u) \leq W_{M}, \quad \text { for every } \quad 0<r \leq 1 / 2 .
$$

Next, consider the rescaled functions

$$
v_{j}(x):=u_{r_{j}}(x), \quad x \in B_{1 / r_{j}} .
$$

Given $K>1$, observe that

$$
\delta\left(r_{j} / 2, u_{K}\right)=\delta\left(r_{j} K / 2, u\right) \geq \delta\left(r_{j} / 2, u\right) / K \geq \epsilon / K
$$

and

$$
W\left(r_{j}, u_{K}\right)=W\left(K r_{j}, u\right) \leq W_{M}
$$


provided $K r_{j} \leq 1 / 2$. Applying now Lemma 5 , we obtain that there exists a constant $C_{\epsilon, M, K}$ such that

$$
\left\|u_{K}\right\|_{L^{\infty}\left(B_{r_{j} / 2}\right)} \leq C_{\epsilon, M, K} r_{j}^{2} \quad \text { for } \quad j \geq j_{\epsilon, M, K} .
$$

This is equivalent to

$$
\|u\|_{L^{\infty}\left(B_{K r_{j} / 2}\right)} \leq C_{\epsilon, M, K} K^{2} r_{j}^{2} \quad \text { for } \quad j \geq j_{\epsilon, M, K}
$$

or

$$
\left\|v_{j}\right\|_{L^{\infty}\left(B_{K}\right)} \leq C_{\epsilon, M, K} K^{2} \quad \text { for } j \geq j_{\epsilon, M, K} .
$$

This means we can extract a subsequence of $v_{j}$ converging to a global solution $v_{0}$ in $C_{\text {loc }}^{1, \alpha}\left(\mathbb{R}^{n}\right)$ (which might not necessarily have a quadratic growth at infinity).

Observe then

$$
W\left(r ; v_{0}\right)=\lim _{j \rightarrow \infty} W\left(r ; v_{j}\right)=\lim _{j \rightarrow \infty} W\left(r r_{j} ; u\right)=W(0+; u) .
$$

In particular, $W\left(r, v_{0}\right)=$ const. Since $v_{0}$ is a solution of (1) with $f=1$, this is possible iff $v_{0}$ is homogeneous of degree two. Then by the classification of such solutions (see Lemma 3), $v_{0}$ is either a halfspace solution or a nonzero homogeneous quadratic polynomial. Let us show that the latter is impossible. Indeed, we have

$$
\delta\left(1 / 2, v_{j}\right)=\delta\left(r_{j} / 2, u\right) \geq \epsilon>0,
$$

which implies that

$$
\delta\left(1 / 2, v_{0}\right) \geq \epsilon .
$$

Thus, if $v_{0}$ were a quadratic polynomial it would have been identically 0 , which would contradict the nondegeneracy (see Lemma 1). Consequently, $v_{0}$ is a halfspace solution. But then

$$
W(0+; u)=W\left(1 ; v_{0}\right)=A_{n},
$$

which proves (33). Once we have both (32) and (33), we can find $r_{j}>0$ as small as we like such that

$$
\delta\left(r_{j}, u\right)>\epsilon \quad \text { and } \quad W\left(r_{j} ; u\right)<2 A_{n}-\epsilon .
$$

Then, applying Theorem A, we complete the proof of the theorem.

\section{REFERENCES}

[ACF84] H. Alt, L. Caffarelli, and A. Friedman, Variational problems with two phases and their free boundaries, Trans. Amer. Math. Soc. 282 (1984), no. 2, 431-461.

[Bla01] I. Blank, Sharp results for the regularity and stability of the free boundary in the obstacle problem, Indiana Univ. Math. J. 50 (2001), no. 3, 1077-1112.

[Bla04]_, Eliminating mixed asymptotics in obstacle type free boundary problems, Comm. Partial Differential Equations 29 (2004), no. 7-8, 1167-1186.

[BS03] I. Blank and H. Shahgholian, Boundary regularity and compactness for overdetermined problems, Ann. Sc. Norm. Super. Pisa Cl. Sci. (5) 2 (2003), no. 4, 787-802.

[Caf77] L. Caffarelli, The regularity of free boundaries in higher dimensions, Acta Math. 139 (1977), no. 3-4, 155-184.

[Caf80] Compactness methods in free boundary problems, Comm. Partial Differential Equations 5 (1980), no. 4, 427-448.

[Caf98] _ , The obstacle problem revisited, J. Fourier Anal. Appl. 4 (1998), no. 4-5, 383402.

[CJK02] L. Caffarelli, D. Jerison, and C. Kenig, Some new monotonicity theorems with applications to free boundary problems, Ann. of Math. (2) 155 (2002), no. 2, 369-404.

[CKS00] L. Caffarelli, L. Karp, and H. Shahgholian, Regularity of a free boundary with application to the Pompeiu problem, Ann. of Math. (2) 151 (2000), no. 1, 269-292. 
[CPS04] L. Caffarelli, A. Petrosyan, and H. Shahgholian, Regularity of a free boundary in parabolic potential theory, J. Amer. Math. Soc. 17 (2004), no. 4, 827-869 (electronic).

[CSS04] L. Caffarelli, J. Salazar, and H. Shahgholian, Free-boundary regularity for a problem arising in superconductivity, Arch. Ration. Mech. Anal. 171 (2004), no. 1, 115-128.

[CS04] L. Caffarelli and H. Shahgholian, The structure of the singular set of a free boundary in potential theory, Izv. Nats. Akad. Nauk Armenii Mat. (2004).

[CGK00] S. Chanillo, D. Grieser, and K. Kurata, The free boundary problem in the optimization of composite membranes, Differential geometric methods in the control of partial differential equations (Boulder, CO, 1999), Contemp. Math., vol. 268, Amer. Math. Soc., Providence, RI, 2000, pp. 61-81.

[Isa90] V. Isakov, Inverse source problems, Mathematical Surveys and Monographs, vol. 34, American Mathematical Society, Providence, RI, 1990.

[KS99] L. Karp and H. Shahgholian, Regularity of a free boundary problem, J. Geom. Anal. 9 (1999), no. 4, 653-669.

[Mar95] A. Margulis, The moving boundary problem of potential theory, Adv. Math. Sci. Appl. 5 (1995), no. 2, 603-629.

[MW05] R. Monneau and G. Weiss, An unstable elliptic free boundary problem arising in solid combustion, preprint, 2005.

[Sha03] H. Shahgholian, $C^{1,1}$ regularity in semilinear elliptic problems, Comm. Pure Appl. Math. 56 (2003), no. 2, 278-281.

[Sha92] H. Shapiro, The Schwarz function and its generalization to higher dimensions, University of Arkansas Lecture Notes in the Mathematical Sciences, 9, John Wiley \& Sons Inc., New York, 1992.

[Ste70] E. Stein, Singular integrals and differentiability properties of functions, Princeton Mathematical Series, No. 30, Princeton University Press, Princeton, N.J., 1970.

[Wei99] G. Weiss, A homogeneity improvement approach to the obstacle problem, Invent. Math. 138 (1999), no. 1, 23-50.

[Wei01] _ An obstacle-problem-like equation with two phases: pointwise regularity of the solution and an estimate of the Hausdorff dimension of the free boundary, Interfaces Free Bound. 3 (2001), no. 2, 121-128.

Department of Mathematics, Purdue University, West Lafayette, IN 47907, USA

E-mail address: arshak@math.purdue.edu

Depatment of Mathematics, Royal Institute of Thechnology, 10044 Stockholm, SweDEN

E-mail address: henriksh@math.kth.se 